\title{
Supplemental fish oil decreases urinary excretion of a marker of bone resorption in healthy adults
}

\author{
Eric E Noreen ${ }^{*}$, Josef Brandauer, Megan H MacNabb \\ From International Society of Sports Nutrition: 8th Annual ISSN Conference and Expo \\ Las Vegas, NV, USA. 24-25 June 2011
}

\section{Background}

Incorporation of fish oil (FO) into the diet of rodents has been shown to result in positive changes in bone health. Currently it is poorly understood if FO has the same effects on bone health in humans. The purpose of this study was to determine the effects of supplemental FO on levels of urinary $\mathrm{N}$-terminal cross-linked telopeptide (NTx), which is a marker of bone breakdown, and how this is related to the morning levels of salivary cortisol and urinary excretion of interleukin 6 (IL-6).

\section{Methods}

A total of twenty-eight females and twelve males(35 \pm $13 y r s ; 69.1 \pm 14.1 \mathrm{~kg} ; 29.4 \pm 9.2 \%$ body fat; mean \pm SD) participated in this study. All testing was conducted in the morning following an overnight fast. Baseline measurements of salivary cortisol were collected via passive drool and baseline measurements of urinary NTxand IL6 were collected from the second void of the day and corrected for creatinine excretion. After baseline testing, subjects were assigned randomly in a double blind manner to one of two groups: $4 \mathrm{~g} / \mathrm{d}$ of Safflower Oil (SO) or $4 \mathrm{~g} / \mathrm{d}$ of FO supplying $1,600 \mathrm{mg} / \mathrm{d}$ eicosapentaenoic acid (EPA) and $800 \mathrm{mg} / \mathrm{d}$ docosahexaenoic acid (DHA). All tests were repeated following $6 \mathrm{wk}$ of treatment. A treatment by time, repeated measures ANOVA was used to evaluate differences between groups over time, and a standard Pearson's $r$ was used to evaluate correlations. Additionally, within group pre-post differences were evaluated using a repeated measures t-test. For all analysis, the alpha level was set at $\mathrm{p}<0.05$.

* Correspondence: enoreen@gettysburg.edu

Department of Health Sciences. Gettysburg College, Gettysburg PA, USA

\section{Results}

Compared to the SO group, there was a significant decrease in urinary creatinine corrected NTx excretion following FO treatment $(\mathrm{SO}=17.5 \pm 42.9 \mathrm{BCE} / \mathrm{mM}$; $\mathrm{FO}=-11.3 \pm 27.7 \mathrm{BCE} / \mathrm{mM} ; \mathrm{p}=0.02)$. There was also a tendency for urinary creatinine corrected IL- 6 excretion $(\mathrm{SO}=-0.08 \pm 1.18 \mathrm{pg} / \mathrm{mg} ; \mathrm{FO}=-1.8 \pm 3.8 \mathrm{pg} / \mathrm{mg} ;$ $\mathrm{p}=0.08)$, and salivary cortisol $(\mathrm{SO}=0.029 \pm 0.283 \mu \mathrm{g} / \mathrm{dL}$; $\mathrm{FO}=-0.069 \pm 0.144 \mu \mathrm{g} / \mathrm{dL} ; \mathrm{p}=0.13)$ to decrease following FO treatment. When analyzed independently, however, there was a significant pre-post reduction for salivary cortisol in the FO group $(\mathrm{p}=0.04)$, with no change in the $\mathrm{SO}$ group $(\mathrm{p}=0.68)$, as well as a significant reduction pre-post for urinary IL- 6 in the FO group $(\mathrm{p}=0.05)$, with no change in the SO group $(\mathrm{p}=0.78)$. However, the change in urinary NTx concentrationwas not related to the change insalivary cortisol concentration $(\mathrm{r}=-0.017, \mathrm{p}=0.9)$, or the change in urinary IL-6 concentration $(\mathrm{r}=-0.323, \mathrm{p}=0.26)$.

\section{Conclusions}

Six weeks of supplementation with FO in adults significantly decreased urinary NTx excretion, but this change was not related to changes in cortisol or IL-6.

\section{Funding}

Gettysburg College Research and Professional Development Grant and Genuine Health Corporation.

Published: 7 November 2011
doi:10.1186/1550-2783-8-S1-P14

Cite this article as: Noreen et al:: Supplemental fish oil decreases

urinary excretion of a marker of bone resorption in healthy adults.

Journal of the International Society of Sports Nutrition 2011 8(Suppl 1):P14. 